\title{
ERROR BEHAVIOR OF ATOMIC CLOCKS ABOARD GPS SATELLITES
}

\author{
Gustavo Bento Mansur ${ }^{1}$ - ORCID: 0000-0003-2963-4886
}

Luiz Danilo Damasceno Ferreira² - ORCID: 0000-0002-8247-313X

${ }^{1}$ Universidade Federal do Paraná, Departamento de Geomática, Curitiba - PR, Brasil.

E-mail: gustavobmansur@gmail.com

${ }^{2}$ Universidade Federal do Paraná, Departamento de Geomática, Curitiba - PR, Brasil.

E-mail: luizdaniloferreira@gmail.com

Received in $24^{\text {th }}$ September 2019

Accepted in $06^{\text {th }}$ October 2019

\section{Abstract:}

The signal transmission and position quality obtained by GNSS positioning directly depends on the time control. The atomic clocks aboard the satellites are responsible for this control. In this research, the satellites clock errors of GPS are studied, using data from the pseudorange, the satellite semi-major axis and the correction parameters of the satellites' clock errors found in the RINEX navigation and observation files. From these data, the satellites' clock errors are calculated using the IGS mathematical model for rubidium and cesium clocks, and then an adjustment technique is applied in order to estimate the new parameters of the clock corrections. For cesium atomic clocks, the periodic part of the mathematical model was adapted. The correction parameters adjusted were applied in the IGS and the adapted model; finally, the results were compared with the IGS data from the clk_30s file. The experiments carried out show an improvement of $50 \mathrm{~cm}$ in the cesium satellites' clock errors. In addition, the research concludes that the satellites' clock errors do not depend on the station where the data were collected and the equipment's antenna.

Keywords: Satellites positioning, broadcast ephemerides, clock error, prediction model.

How to cite this article: MANSUR, G. B.; FERREIRA, L. D. D. Error behavior of atomic clocks aboard GPS SATELLITES. Bulletin of Geodetic Sciences. 25(4): e2019027, 2019. 


\section{Introduction}

The GNSS (Global Navigation Satellite System) is currently used in a broad range of activities as point positioning and navigation that require high precision. The measured quality of a point is directly related to orbit solutions, the troposphere and ionosphere, the multipath, the phase center of the antenna and the satellite/receptor clock errors (Bidikar et al., 2014; Senior et al., 2008; Seeber, 2003).

Over the years the evolution of atomic clocks brought innovations to metrology, fundamental physics and global positioning techniques (Hinkley, 2013; Delva and Lodewyck, 2013). The positioning using GNSS satellites depends on the propagation time that the signal takes to travel from the satellite to the receiver. Therefore, an error of time implies an error of position; thus, navigation systems depend on clock performances and the ability to estimate their behavior (Seeber, 2003; Martinez, 2014; Galleani, 2014). The atomic clocks are used not just on satellites but also on terrestrial stations, where they can synchronize clocks that are in orbit and characterize the time systems (Galleani, 2014).

Atomic clocks that are in orbit suffer from anomalies such as radiation, temperature, aging, relativistic effects and even a sudden stoppage of work. Understanding the anomalies that influence the atomic clocks is essential for applying adequate corrections to the positioning. The IGS (International GNSS Service) is the main organization responsible for orbit and clock product information (Galleani, 2014).

The GPS satellites carry on-board atomic clocks of rubidium and cesium, which have accuracies on the order of $10^{-9}$ and $5 \times 10^{-13}$ seconds, respectively (Teunissen and Montenbruck, 2017). Despite the high stability of the clocks, differences in synchronization still exist between the time transmitted by the satellite and the GPS time. Therefore, it is necessary to provide correction of the satellite clock error (Seeber, 2003). This error is computed from a second-degree polynomial function individually for each satellite (Ray, 2001; LV et al., 2017). In addition, the correction factor is common to every user; in other words, users that are in different positions observing the same satellite are susceptible to the same error (Bidikar et al., 2014).

Diverse methodologies have been studied aiming to improve the clock prediction models as well in the work of Huang (2013), Hauschild and Montenbruck (2008), where a Kalman filter was used to determinate the satellite clock error. More recently, there are studies focused on the clocks' improvements for real time positioning as in the research of Strandjord and Axelrad (2018) where a determination of a sub daily set of the clock parameters is applied.

In this following research we focus on apply a parametric adjustment in order to determine new clock parameters and hence compute the errors with these parameters. Moreover, we adapted the periodic part of the mathematical model that determines the clock errors for satellites with cesium atomic clock, adding an exponential function. The results show an approximation of the values computed in the study according with the IGS as reference, especially for the cesium clocks.

\section{Theory Review}

The following section briefly address the theory about the mathematical modeling of the satellite clock errors.

\subsection{Clocks and frequency standards}

To obtain reliable information about time, stable clocks are necessary; thus, the frequency oscillation needs Bulletin of Geodetic Sciences, 25(4): e2019027, 2019 
to attain a stability standard over time. The atomic clocks are responsible for reliable information, and the most important equipment inside the clock is the oscillator. Using observations from the oscillator cycles, electronic equipment performs the transformation to the seconds unit.

According to Seder (2008), the performance of a frequency source is defined according to its stability and accuracy, where accuracy is the capacity of the frequency source to be synchronized with another specific frequency (standard frequency). With this concept, it is possible to calculate the frequency deviation from equation 2.1:

$$
\Delta f=f-f_{0},
$$

where:

$f$ - frequency source,

$f_{0}$-standard frequency.

In an ideal concept, the frequency adopted by a clock should never change. However, it is impossible to obtain this stability due to systematic and random errors in the clock components. The environment also contributes to the frequency instability. Thus, the frequency behavior is usually described by the following equation (Seder, 2008):

where:

$$
f(t)=f_{0}+\Delta f+\left(t-t_{0}\right) \dot{f}+\tilde{f}\left(t-t_{0}\right),
$$

$\Delta f$ - frequency bias;

$\dot{f}$ - frequency drift;

$\tilde{f}$ - random frequency error;

$t_{0}$ - initial reference epoch;

$t$ - random reference epoch.

From equation 2.2, using algebraic operations, as demonstrated on Seeber (2003) we reach the equation 2.3, which represents the clock synchronization error:

where:

$$
\Delta t_{i}=T_{i}\left(t_{0}\right)+R_{i}\left(t-t_{0}\right)+\frac{D_{i}}{2}\left(t-t_{0}\right)^{2}+\int_{t 0}^{t} y(t) d t
$$

$T_{i}\left(t_{0}\right)$ - constant time bias,

$R_{i}$ - time drift,

$D_{i}$ - quadratic term (drift rate, aging),

$y(t)$ - random relative frequency error.

Therefore, following the work of Seeber (2003) and Seder (2008), it is possible to obtain the GPS satellite clock error equation 2.4 , changing the terms:

$$
\begin{aligned}
T_{i} & =a_{0} \\
R_{i} & =a_{1} \\
\frac{D_{i}}{2} & =a_{2}
\end{aligned}
$$

thus,

$$
\delta t^{s}(t)=a_{0}+a_{1} \cdot\left(t-t_{0}\right)+a_{2} \cdot\left(t-t_{0}\right)^{2}
$$

where:

$a_{0}-$ clock bias, 
$a_{1}$ - clock drift,

$a_{2}$ - frequency drift (aging).

To determine equation 2.4 the terms $a_{0}, a_{1}$ e $a_{2}$ must be estimated; thus, the clock error depends directly of these parameters. Data from stations spread around the globe are used to compare the parameter values, helping in the detection of random errors and obtaining a reliable initial estimate of the parameters.

\subsection{GPS transmission signal}

The GPS time is characterized by the atomic clocks contained in the GPS system. The clocks that maintain the time scale stand in the central control station on the earth's surface (Seeber, 2003). The clocks aboard satellites should correspond to the GPS time; however, this does not happen because there are errors in the clock oscillator. The signal transmission errors are measured in nanoseconds, meaning position errors are on the order of $30 \mathrm{~cm}$ (Huang et al., 2013). Therefore, a correction must be done, providing a compatibility with the satellite transmission signal and the GPS time scale. To do this, the following equation is used (Bidikar et al., 2014):

$$
t_{G P S}^{t}=t_{t}^{S}-\delta t^{S}
$$

where:

$t_{G P S}^{t}$ - corrected GPS time of transmission,

$t_{t}^{S}$ - satellite time transmission,

$\delta t^{s}$ - satellite clock error.

For the GPS satellites, equation 2.4 is rewritten as (Monico, 2008; Seder, 2008):

$$
\delta t^{S}(t)=a_{0}+a_{1} \cdot\left(t_{G P S}-t_{o c}\right)+a_{2} \cdot\left(t_{G P S}-t_{t o c}\right)^{2}
$$

With $t_{o c}$ as the reference time for the coefficients, then, the term $t_{G P S}$ in equation 2.6 can be replaced by without compromising the quality of the results knowing that is an iterative process, hence first uses as an approximation to compute $t_{G P S}$, and consequently update the value until achieve the convergency. (Monico, 2008; Gemael and Andrade, 2004):

$$
\delta t^{S}(t)=a_{0}+a_{1} \cdot\left(t_{t}^{S}-t_{o c}\right)+a_{2} \cdot\left(t_{t}^{S}-t_{t o c}\right)^{2},
$$

From the pseudorange equation (Seeber, 2003; Bidikar et al., 2014):

$$
P R=c\left(t_{r}-t_{G P S}^{T}\right)
$$

it is possible, with a good approximation, to calculate the satellite transmission signal, using equation 2.8.1 (Bidikar et al., 2014; Seeber, 2003):

$$
t_{G P S}^{t}=t_{r}-\frac{P R}{C}
$$

where $t_{r}$ is the satellite reception signal in the satellite frame, $P R$ is the pseudorange and $c$ is the speed of light in a vacuum. Then, equation 2.7 is written as:

$$
\delta t^{s}(t)=a_{0}+a_{1} \cdot\left(t_{G P S}^{t}-t_{o c}\right)+a_{2} \cdot\left(t_{G P S}^{t}-t_{t o c}\right)^{2}
$$

Remarking that the satellite clock parameters are given by the control center as an ionosphere-free combination, the user must take care if only working with single frequencies (Kwan, 2019). In the work presented we used the parameters as they are distributed in the RINEX file. Another remark is that the relativistic effects were not considered on the experiments. 


\section{Experiments}

For the experiments, we have chosen four stations distributed from south to north of Brazil and these stations are part of the Continuous Monitoring Brazilian Network from the IBGE (Brazilian Institute of Geography and Statistics), shown in Table 1. Only GPS satellites in the interval of June 26, 2016 until July 03, 2016 were used.

Table 1: RBMC Stations

\begin{tabular}{c|c|c}
\hline Station & City/State & Antenna \\
\hline UFPR & Curitiba-PR & ZEPHYR GNSS GEODETIC MODEL 2 (TRM55971.00) \\
\hline RECF & Recife-PE & GNSS CHOKE RING (TRM59800.00) \\
\hline SMAR & Santa Maria- RS & GNSS CHOKE RING (TRM59800.00) \\
\hline MSCG & Campo Grande-MS & ZEPHYR GNSS GEODETIC MODEL 2 (TRM55971.00) \\
\hline
\end{tabular}

As first step we computed the satellite clock errors using the parameters provided in the RINEX files from the stations. These errors entered as observations in the adjustment further applied. We followed different mathematical models according to the element in the oscillator. First for satellites with rubidium, equation 2.9 was used, rewritten as:

$$
\delta t^{s}(t)=a_{0}+a_{1} \Delta t+a_{2} \Delta t^{2}
$$

where $\Delta t$ is the time difference.

The values of $a_{\sigma^{\prime}} a_{1}$ and $a_{2}$ were read directly from the RINEX navigation file. For satellites with cesium, the complete satellite clock error equation with the linear part was used, with the periodic term, as in equation 3.1 (Seder 2008; Huang et al. 2013).

$$
\delta t^{s}=a_{0}+a_{1} \Delta t+a_{2} \Delta t^{2}+A_{0} \operatorname{sen}(\omega \Delta t+\phi)+\varepsilon,
$$

$A_{0}$ is the amplitude, $\omega$ is the angular frequency, $\phi$ is the phase shift, and $\varepsilon$ is the noise. For the amplitude values, we adopted the research of Martinez (2014), that presents on his work a robust study about how the amplitude have strong impact in the clock prediction. The phase shift $(\phi)$ is calculated for each satellite (Brodebauer And Weber, 2004; Seeber, 2003); thus, the satellite clock error of the cesium element can be calculated.

A parametric least square method was applied in order to define new values for $\frac{\partial \delta t^{s}}{\partial a_{1}}=\Delta t$ and $\frac{\partial \delta t^{s}}{\partial a_{1}}=\Delta t$, since these are the parameters with major influence in the clock offset (Martinez, 2014) and the value of $a_{2}$ is zero for the entire track time in the navigation RINEX file we opted for only use those two parameters in our adjustment. With the clock error calculated previously from the coefficients in the RINEX file, the observations $\left(L_{b}\right)$ vectors were created and the Jacobian matrix is composed of and, where the matrix with the dimension, respectively, depends on how many times the parameters change their values in the navigation file and the number of observations for the satellite. Equation 3.2 shows how the Jacobian matrix was assembled.

$$
A=\left[\begin{array}{cccccc}
1 & \Delta t_{1} & 0 & 0 & \cdots & \cdots \\
1 & \Delta t_{2} & 0 & 0 & \cdots & \cdots \\
\vdots & \vdots & 0 & 0 & \cdots & \cdots \\
1 & \Delta t_{n} & 0 & 0 & \cdots & \cdots \\
0 & 0 & 1 & \Delta t_{n+1} & \cdots & \cdots \\
0 & 0 & 1 & \Delta t_{n+2} & \cdots & \cdots \\
0 & 0 & \vdots & \vdots & \cdots & \cdots \\
0 & 0 & 1 & \Delta t_{m} & \cdots & \cdots
\end{array}\right],
$$


The weight matrix adopted with size $m \times m$, was the identity matrix since there was no information about the precision of the parameters. The adjustment was calculated for all satellites available on the days of observation. Thus, from the new adjusted parameters, the satellites' clock errors were recalculated.

In order to study how the cesium satellite clock error behaves when the periodic part in the equation changes, we performed using the periodic part of equation 3.1. We tested different combinations of functions including sine and cosine, and among others, the one that showed a satisfactory test result was an exponential function. Hence, we computed the cesium satellite clock errors with the modified model, as shown in equation 3.3:

$$
\delta t^{s}=a_{0}+a_{1} \Delta t+a_{2} \Delta t^{2}+A_{0} \operatorname{sen}\left(\omega \Delta t+e^{\omega \Delta t}+\phi\right)+\varepsilon,
$$

The adjustment results were similar for all satellites, with the value of the residual vector on the order of $10^{-14}$ seconds.

For a better interpretation of the data obtained, graphics were produced to represent the satellites' clocks error behaviors. The next figures represent some of the satellites; PRN8 and PRN24 have a cesium element, and PRN16 and PRN5 with rubidium. To simplify, the errors calculated from the adjusted parameters will be referred to as modified clock errors, while the clock errors obtained directly from RINEX as original clock errors.

In figure 1, from the UFPR Station, it is possible to observe two different behaviors. In the modified and original clock errors, the behavior is practically a constant straight line; that is, the word "practically" is used because the error values of the difference between one reading and the next (30 seconds later) are on the order of $10^{-18}$ seconds, for both data values, modified and original. The main reason for those straight lines appears to be that the correction parameter of satellite clock error (in the RINEX file) is constant for a period of time, for example, from 00 h 00 min 00 s to 1 h 59 min 45 s, and they change "jumping" to a new parameters' values.

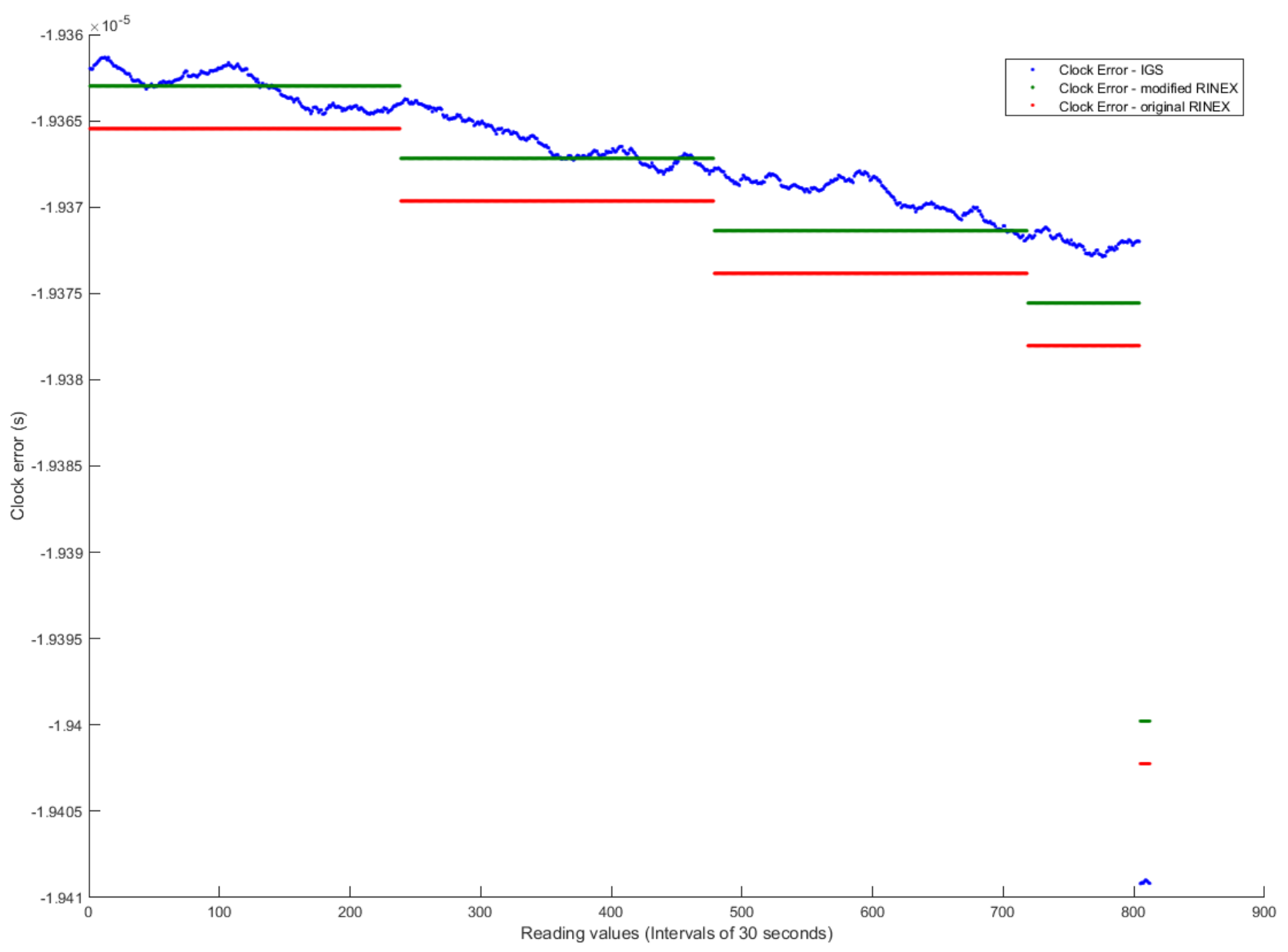

Figure 1: Clock error behavior - PRN24 (CS) - UFPR Station - June 30 of 2016. 


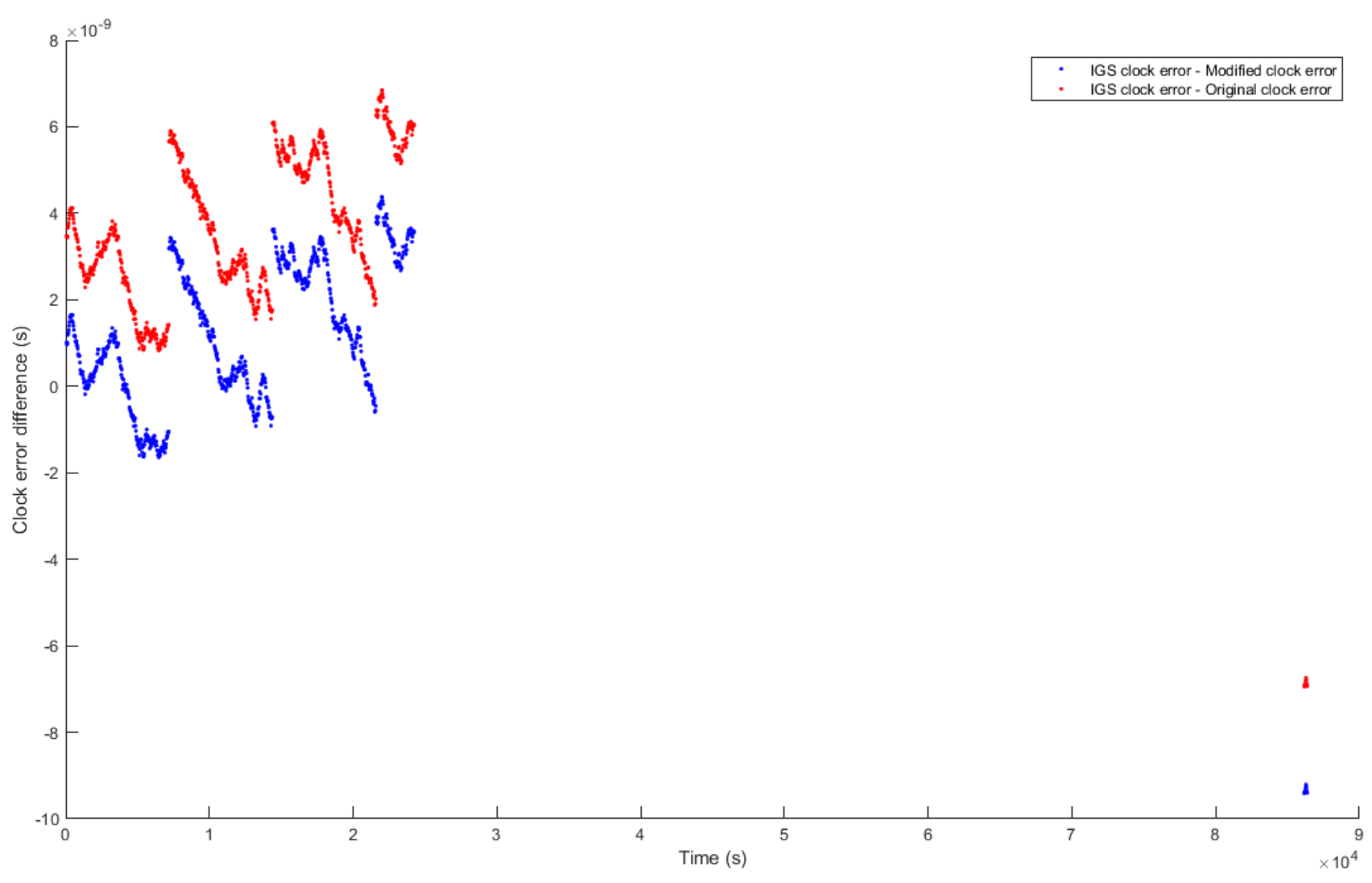

Figure 2: Clock error differences - PRN24 (CS) - UFPR Station - June 30 of 2016.

In figure 2, the differences between the modified and the IGS clock errors are presented, as are the original and the IGS clock errors. The differences shown are on the order of $10^{-9}$ seconds. Analyzing both differences, it is possible to relate that when the adjusted parameter was used to calculate the clock error, the result presented was closer to zero; i.e., the values were closer to the IGS results. In addition, there is a space in figure 2 that does not contain points, indicating no GPS signal was present on this interval. In order to verify the same satellite behavior, we checked the values using the MSCG station, on June 30 of 2016. As expected, the satellite clock error behavior in figure 3 is similar to that of the UFPR station (figure 1), where the modified clock error approaches to the IGS error. The differences, shown in figure 4, tend to follow the same behavior as in figure 2.

Regarding the PRN8 (June 28 of 2016) in the figures 5 and 6, the error behavior was similar to that of PRN24, with the presence of the straight lines and the "jump" every time the corrections parameters changed, and the values of the errors were also on the order of $10^{-9}$ seconds.

For satellites with rubidium in the oscillator the adjusted parameters and the originals had a difference on the order of $10^{-17}$ seconds. As expected, the clock error of the satellites with rubidium showed a behavior that followed the same shape of the satellites with cesium, where the difference between the IGS errors and the error with adjusted parameters is next to zero in the beginning and increases over time. This behavior is repeated when the parameters change. Also, with rubidium, the satellite PRN16 was evaluated, from on July 02 of 2016, for the SMAR and UFPR Stations, and the behavior between the two stations was similar. The difference errors (IGS - adjusted parameters) are shown in the figure 7. We verified the clock error among all monitoring stations using data from one satellite using the PRN05, on July 01 of 2016. From figure 8, it is possible to identify a continuity of the errors, showing that the clock error does not depend of station or antenna model, and there is overlapping when signal tracking exists on two or more stations. 


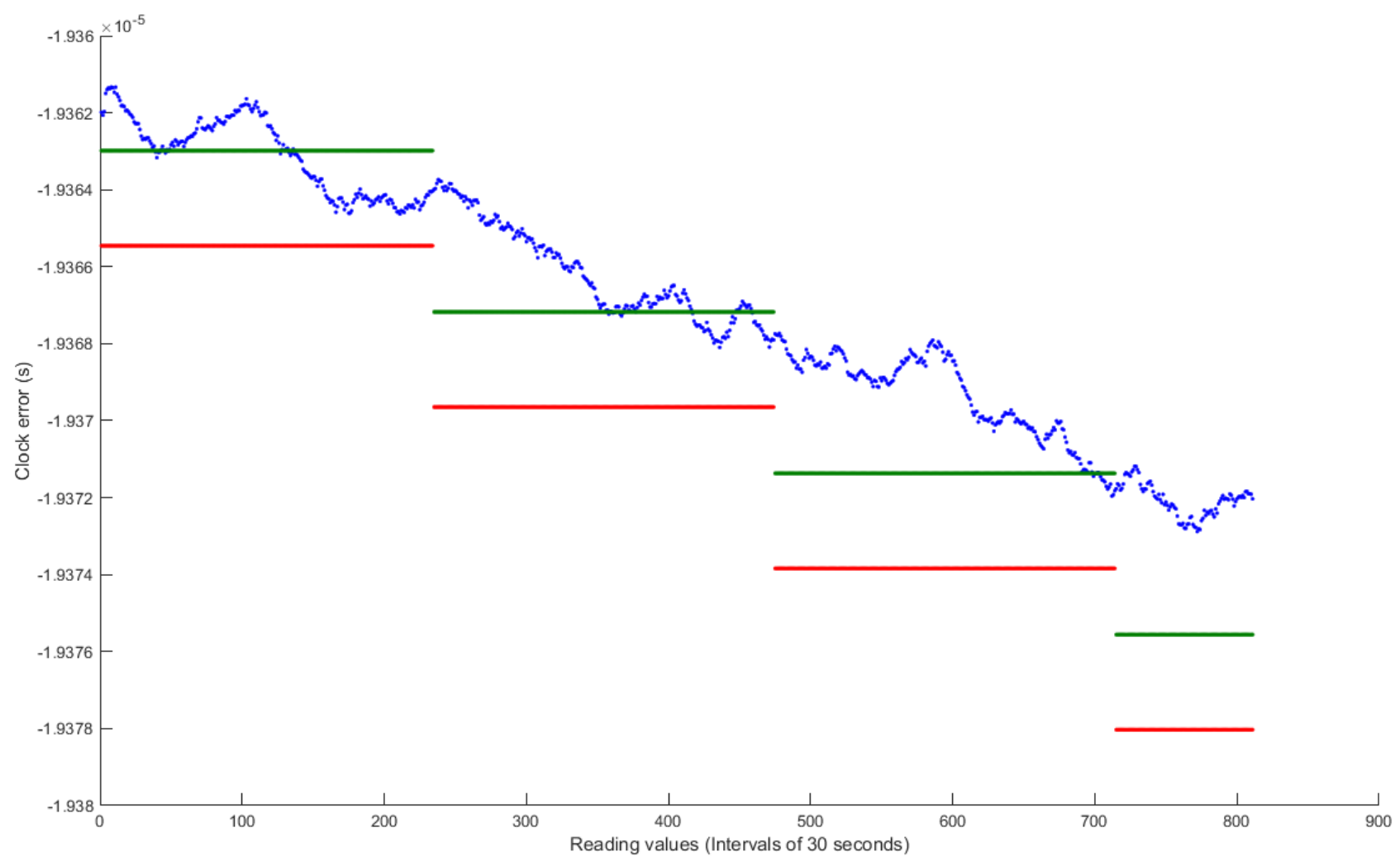

Figure 3: Clock error behavior - PRN24 (Cs) - MSCG Station - June 30 of 2016.

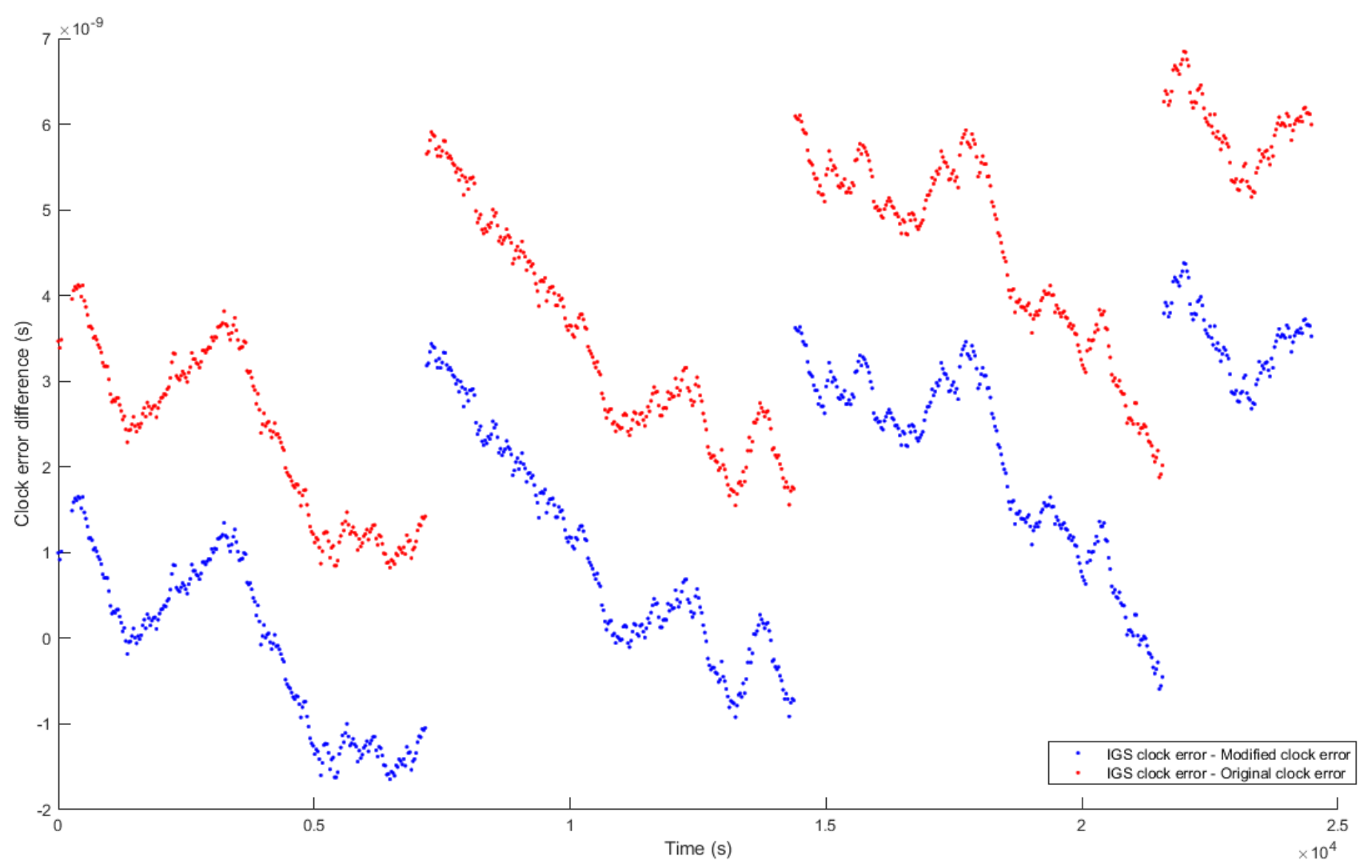

Figure 4: Clock error differences - PRN24 (CS) - MSCG Station - June 30 of 2016. 


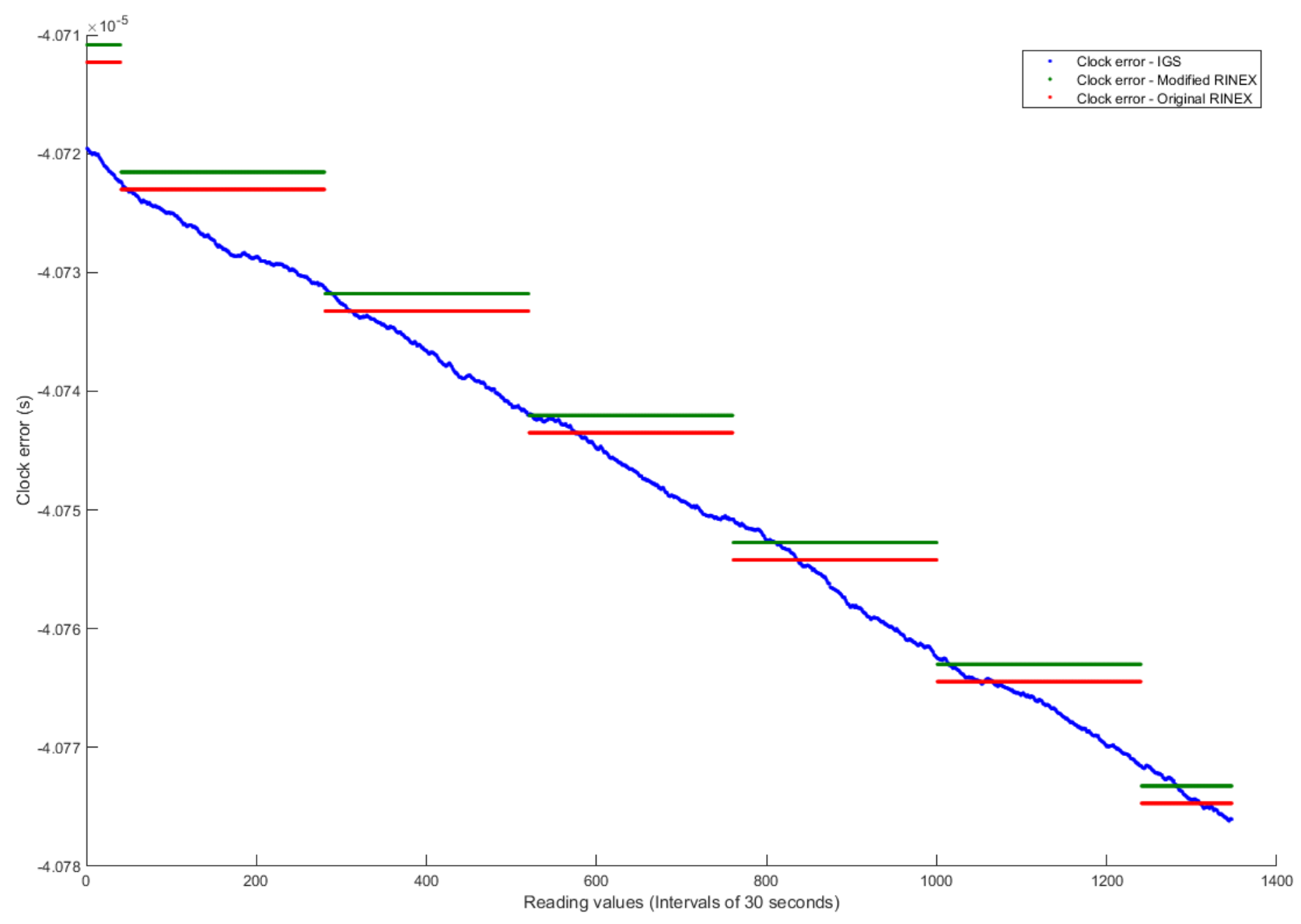

Figure 5: Clock error behavior - PRN8 (Cs) - RECF Station - June 28 of 2016.

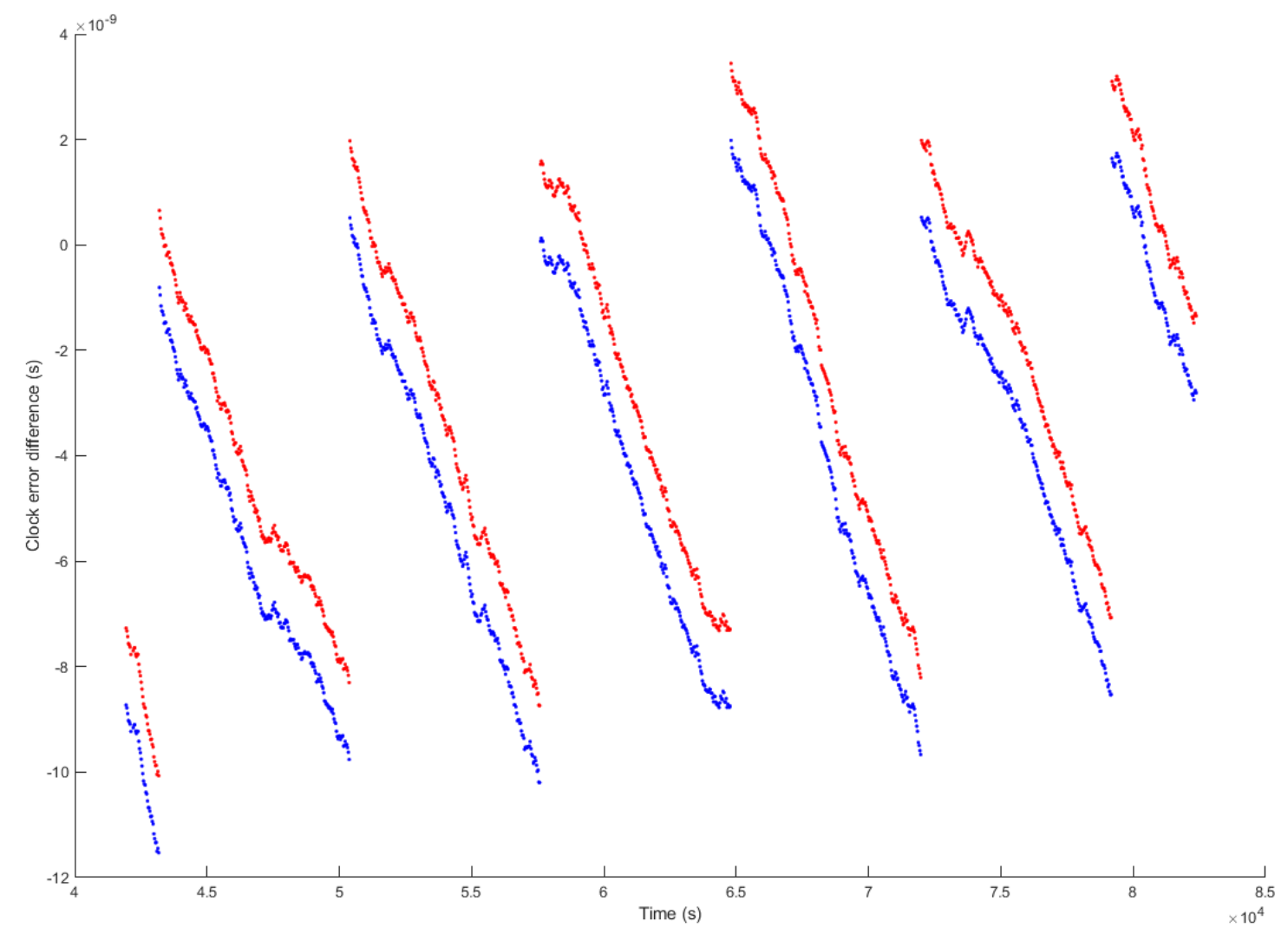

Figure 6: Clock error differences - PRN8 (CS) - RECF Station - June 28 of 2016. 


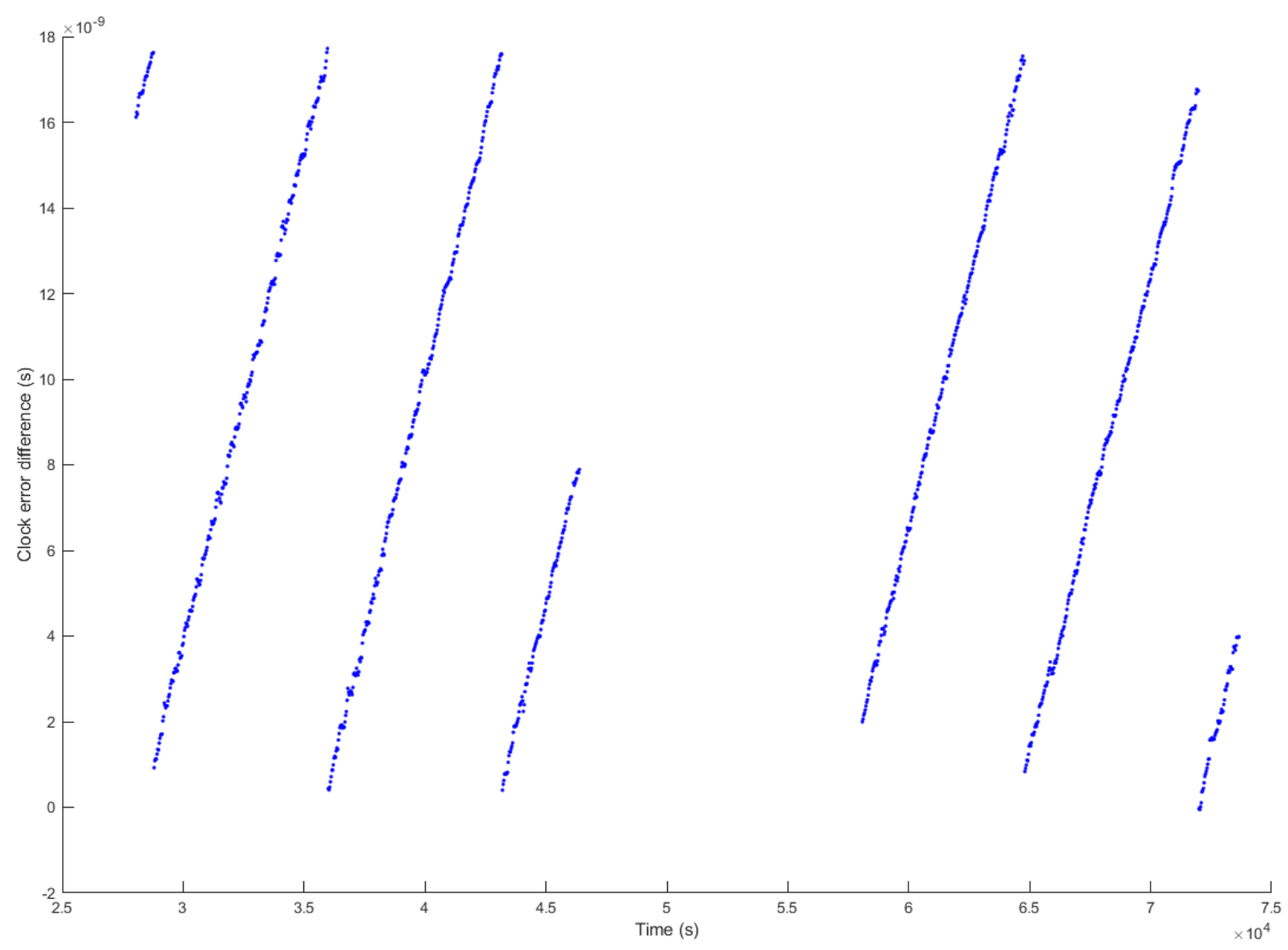

Figure 7: Clock errors differences - PRN16 (Rb) - SMAR Station - July 02 of 2016.

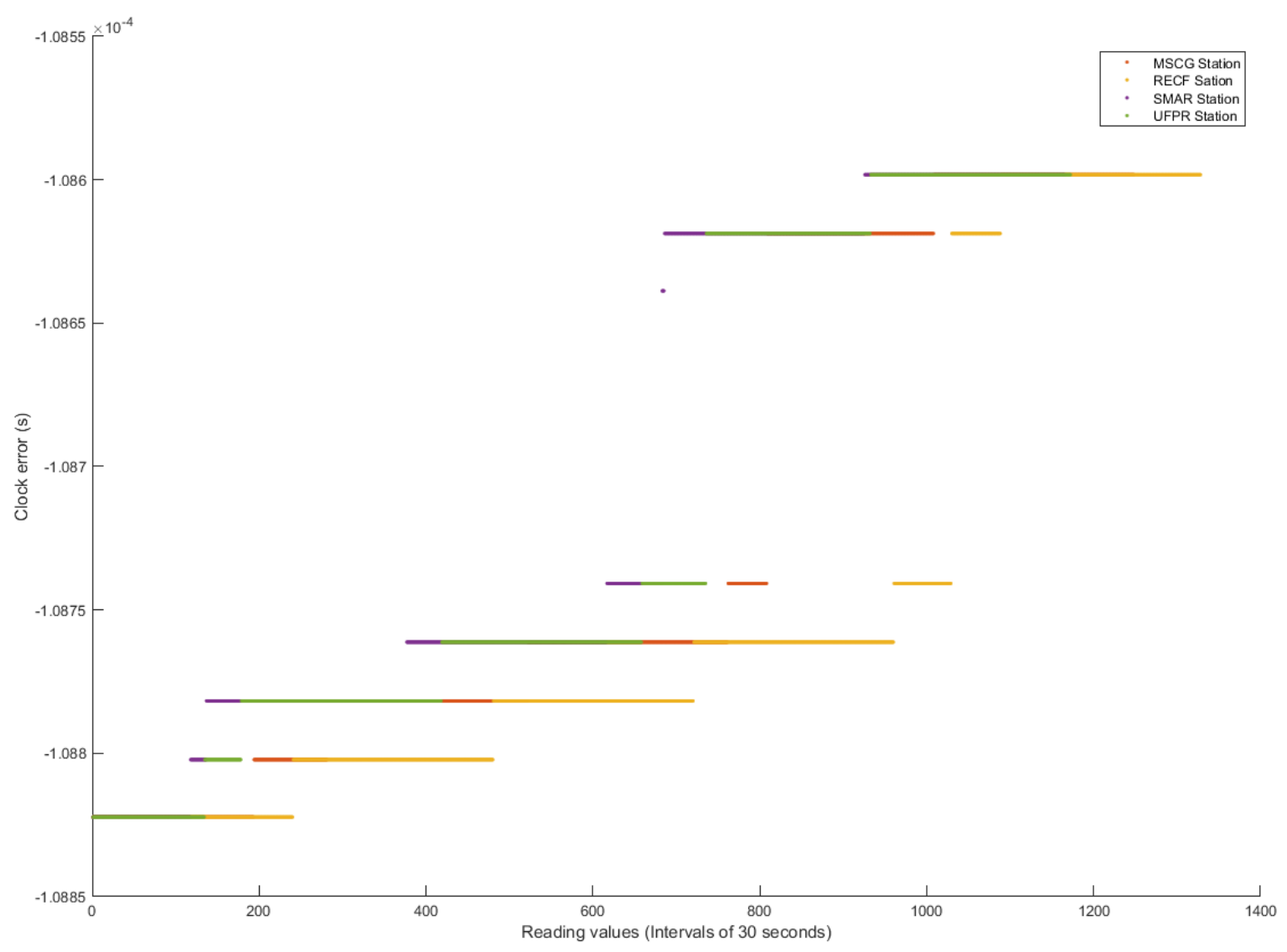

Figure 8: Modified clock errors - PRN05 - Stations: UFPR/RECF/SMAR/MSCG - July 01 of 2016. 
Table 2: Satellite clock errors comparison.

\begin{tabular}{c|c|c|c}
\hline \multicolumn{4}{|c}{ Satellite $16(\mathrm{Rb})$} \\
\hline Station & Mean $(\mathrm{s})$ & Standard Deviation $(\mathrm{s})$ & RMS $(\mathrm{s})$ \\
\hline UFPR & $-9,57294 \times 10^{-9}$ & $4,73978 \times 10^{-9}$ & $1,07 \times 10^{-8}$ \\
\hline SMAR & $-9,41635 \times 10^{-9}$ & $4,86096 \times 10^{-9}$ & $1,0597 \times 10^{-8}$ \\
\hline RECF & $-1,02921 \times 10^{-8}$ & $5,00278 \times 10^{-9}$ & $1,14435 \times 10^{-8}$ \\
\hline MSCG & $-9,90569 \times 10^{-9}$ & $4,88324 \times 10^{-9}$ & $1,10439 \times 10^{-8}$ \\
\hline \multicolumn{5}{|c}{ Satellite $24(\mathrm{Cs})$} \\
\hline UFPR & $-1,13255 \times 10^{-9}$ & $1,85825 \times 10^{-9}$ & $2,17618 \times 10^{-9}$ \\
\hline SMAR & $-5,48227 \times 10^{-10}$ & $2,86201 \times 10^{-9}$ & $2,91405 \times 10^{-9}$ \\
\hline RECF & $-1,60315 \times 10^{-9}$ & $1,50375 \times 10^{-9}$ & $2,19803 \times 10^{-9}$ \\
\hline MSCG & $-1,26728 \times 10^{-9}$ & $1,56066 \times 10^{-9}$ & $2,01039 \times 10^{-9}$
\end{tabular}

Table 2 shows the mean error for the satellites PRN16 and PRN24 according to station, as well the standard deviation and the RMS computed between the IGS error and the error calculated using the adjusted parameters obtained by the methodology presented. The RMS regarding the cesium satellite is smaller than the rubidium, showing what we expected, that the cesium is closer to the IGS.

\section{Results analysis and Conclusions}

In this research, 31 satellites currently functional for GPS were evaluated. One important characteristic observed was the presence of the "straight lines" that are on the original RINEX file as well as the adjusted parameters file. This behavior is different from the IGS errors, because according to Martinez (2014), the methodology used by the IGS for an interpolation is applied to the parameters, making then different for each 30 seconds interval, not constant as in the navigation files. It is important to explain that the "jumps", mentioned before, occur because there is a change in the parameters $\left(a_{a^{\prime}} a_{1}, a_{2}\right)$; hence, the clock errors are directly influenced.

The figures $(1,2,3,4,5$ and 6$)$ presented the results of the satellites with cesium and showed that the error calculated using equation 3.3 and the adjusted parameters was closer to the IGS error than the error calculated with the original RINEX. These results indicate that the exponential function added to the IGS equation has a satisfactory usage and which, if applied, can help studies about rapid solutions achieve a better performance.

The satellites with rubidium have a small difference $\left(10^{-17}\right.$ seconds) between the error calculated with adjusted parameters and the original RINEX. Analyzing the difference compared to the IGS error, the same behavior that occurred with the cesium clocks was noticed in the rubidium clocks. Whenever the parameter changed, the difference was closer to zero and increased over time. It was observed that in rubidium clocks, this difference was larger than it was in the cesium clocks, considering when the parameters were constant (approximately 2 hour). In figure 8, the PRN5 was analyzed using data from all stations. The result indicates that the clock error does not depend of the station and antenna model.

The adjustment technique and the modifications done in the equation of the clock corrections presented a good methodology to study the behavior of the error in the atomic clocks aboard satellites. A point of importance of this study was the exponential function added in the periodic part of the equation, where the result is promising and showed that the values differences between the modified RINEX and the IGS offset were small. The function used can also benefit studies that deal with real time positioning since these techniques requires a good modeling of the clock offset. Although the rubidium satellites, as mentioned, do not showed big differences between the adjusted parameters and the originals, it is possible to say that the results were consistent with the IGS. 


\section{AUTHOR'S CONTRIBUTION}

L.F. conceived of the presented idea. G.M. and L.F. developed the theory and G.M. performed the computations. G.M. carried out the experiments and wrote the manuscript with support from L.F. L.F. supervised the findings of this work. All authors discussed the results and contributed to the final manuscript.

\section{References}

BIDIKAR, Bharati et al. Satellite Clock Error and Orbital Solution Error Estimation for Precise Navigation Applications. Positioning. p.22-26. dez. 2013.

BROEDERBAUER, V.; WEBER, R.; Modeling of GPS Satellite Clocks and Comparisons. 10 years IGS, workshop \& Symposium Berne, Suíça, 01-05 de março de 2004.

DELVA, Pacome; LODEWYCK, Jerome. Atomic clocks: new prospects in metrology and geodesy. Paris, out. 2013.

GALLEANI, Lorenzo. Characterizing Changes In The Noise Statistics Of Gnss Space Clocks With The Dynamic Allan Variance. Torino, 2014.

GEMAEL, Camil; ANDRADE, José Bittencourt. Geodésia Celeste. Curitiba: Ufpr, 2004. 389 p.

HAUSCHILD, André; MONTENBRUCK, Oliver. Kalman-filter-based GPS clock estimation for near real-time positioning. GPS Solutions, [s.l.], v. 13, n. 3, p.173-182, 16 nov. 2008. Springer Science + Business Media. http://dx.doi. org/10.1007/s10291-008-0110-3.

HINKLEY, N. et al. An Atomic Clock with 10-18 Instability. Science, p.1215-1218, set. 2013.

HUANG, Guan Wen; ZHANG, Qin; XU, Guo Chang. Real-time clock offset prediction with an improved model. GPS Solutions, Berlin, v. 18, n. 1, p.95-104, 13 fev. 2013. Springer Science + Business Media. http://dx.doi.org/10.1007/ s10291-013-0313-0.

IBGE. Rede Brasileira de Monitoramento Contínuo dos Sistemas GNSS - RBMC. Available in em: <http://www.ibge. gov.br/home/geociencias/geodesia/rbmc/rbmcpesq.shtm?c=9>. Accessed in: 24 mar. 2016.

KWAN, P. (2019). NAVSTAR GPS Space Segment/Navigation User Segment Interfaces. Interface Control.

LV, Y., DAI, Z., ZHAO, Q., YANG, S., ZHOU, J., and LIU, J. (2017). Improved short-term clock prediction method for realtime positioning, Sensors (Switzerland). https://doi.org/10.3390/s17061308.

MARTINEZ, Francisco Javier Gonzalez. Performance of new GNSS satellite clocks. 2014. 228 f. Tese (Doutorado) Curso de Geodatisches Institut, Schriftenreihe Karlsruher Institut Für Technologie, München, 2014.

MONICO, João Francisco Galera. Posicionamento pelo GNSS: Descrição, fundamentos e aplicações. 2. ed. São Paulo: Unesp, 2008. $476 \mathrm{p}$.

RAY, J. Summary of clock prediction strategy, Tech. Rep. (update of IGS Mail \#2962), 2001.

SEDER, Heinz. Study of GPS satellites clock's behaviour. 2008. 22 f. Department Of Physics And Earth Sciences, University of Leipzig, Leipzig, 2008

SEEBER, Günter. Satellite Geodesy. 2. ed. Berlin: Walter de Gruyter, 2003. 589 p.

SENIOR, Kenneth L.; RAY, Jim R.; BEARD, Ronald L. Characterization of periodic variations in the GPS satellite clocks. GPS Solutions, v. 12, n. 3, p.211-225, 14 fev. 2008. Springer Science + Business Media. http://dx.doi.org/10.1007/s10291008-0089-9. 
STRANDJORD, K. L., \& AXELRAD, P. (2018). Improved prediction of GPS satellite clock sub-daily variations based on daily repeat. GPS Solutions, 22(3), 1-13. https://doi.org/10.1007/s10291-018-0723-0

TEUNISSEN, P. J. and MONTENBRUCK, O.: Springer Handbook of Global Navigation Satellite Systems, Springer International Publishing, Cham, https://doi.org/10.1007/978-3-319-42928-1, https://www.hh.se/download/18.70 cf2e49129168da015800078805/10global-navigation-satellite-system.pdf http://link.springer.com/10.1007/978-3319-42928-1, 2017. 